\section{On the category effect in visual search}

\author{
J. A. DEUTSCH \\ University of California, San Diego \\ La Jolla, California 92093
}

When items of one category (e.g., numbers) are placed among items belonging to another category (e.g., letters) they are detected more quickly and accurately than when items in the same category (e.g., letters) are placed among other items (e.g., other letters) in the same category (Brand, 1972; Egeth, Jonides, \& Wall, 1972; Gleitman \& Jonides, 1976; Ingling, 1972; Jonides \& Gleitman, 1972). A possible simple explanation of such an effect could be that the two categories do not share one or more component features and that such unshared features are easier to detect than the unshared features of the items within a category. However, such an explanation seems to be contradicted by a most elegant demonstration by Jonides and Gleitman $(1972,1976)$ and Gleitman and Jonides (1976). These authors have shown that the effect occurs when the same shape belonging to two categories simultaneously is categorized differently, as in the following example. The same shape represents both a "zero" and the letter "oh." If subjects are asked to detect an "oh" among a group of letters, or a "zero" among a group of letters, their performance is better in the second case. The way the identical shape is categorized affects its ease of detection.

However, in spite of such evidence against the "feature" explanation of the categorization effect, Gleitman and Jonides (1976) and Jonides and Gleitman $(1972,1976)$ argue that a version of the feature hypothesis is essentially correct. To quote: "To explain this phenomenon one must assume that the item ' 0 ' is ambiguous in the sense of containing some features of both the letter and digit categories. One must further assume that some facets of the subject's feature extraction mechanism can be guided by instruction and are thus flexible. Thus in searching for a zero among letters, one extracts digit-defining features; in searching for an 'oh' among letters, one examines items for features that define the letter 'oh.' "' (Jonides \& Gleitman, 1976, p. 290). Whether such a hypothesis is correct may be strongly doubted. The hypothesis is logically inadequate and rests on a number of unlikely assumptions.

My thanks are extended to Drs. Diana Deutsch, James L. McClelland, and Jeffrey $O$. Miller for helpful comments and discussion.
The hypothesis assumes that there is a class of digit-defining features shared in common by all digits which is different from the class of letter-defining features shared in common by all letters. This seems quite unlikely. The division of shapes into arabic numerals and letters seem arbitrary, and two of the numerals are identical with three letters $(0,1$, and capital I).

However, let us, for the sake of argument, assume that such shared category-defining features exist. Then we must also assume that the shared categorydefining features of digits are more easily discriminable than the defining features of individual letters, differentiating them from other letters for the following reason. If the feature extraction mechanism is flexible, then, when the subject is asked to detect a letter among other letters, we would expect the subject to examine for features that differentiate that letter from other letters. Each letter is a different category from other letters, and if the subject can select digit-defining features to detect a certain shape among letters, then the subject should also be able to select "oh" or "zee" defining features to define the category of a single letter, excluding those features which all letters supposedly share. There should therefore be no average advantage for "digit among letters" search over "letters among letters" search. For this to be the case, shared category-defining features of digits or of letters should be easier to detect than the defining features of all the individual letters or digits, which can also be regarded as categories.

But is it at all plausible to believe that the features or features that all letters or all digits have in common are somehow easier to detect than those features which set off or differentiate single letters or digits from each other? It is easy to verbalize or describe differences between individual letters and other letters (or between numbers). Generic differences between letters, on the one hand, and numbers, on the other, are not verbally accessible (probably because they are nonexistent). Nevertheless the flexible feature hypothesis, to explain the category effect, would have to assume that such generic features of letters and numbers are, on the average, easier to detect than the features that differentiate single letters or numbers.

The last objection is the most serious. The subject has been instructed to identify an "oh" or a "zero." The Jonides-Gleitman theory arbitrarily assumes that the subject extracts digit or letter-defining features rather than the features of the letter or number which he has been asked to find. A choice of the features of the actual category (oh, zero, A, etc.) 
that the subject has been asked to select would not produce the correct prediction and yet the theory nowhere explains why the subject does not select such a category. Such an explanation would appear to be critical, and without it the theory is logically inadequate.

\section{AN ALTERNATIVE HYPOTHESIS}

(1) The first assumption is that feature detectors can be selectively facilitated by instruction, but only at their connection to motor output and memory storage (Deutsch \& Deutsch, 1963) and not to their connection to other feature detectors. Such facilitation reduces the latency of firing (and increases the rate) at the connection of the feature detector to the motor output of the detector and improves the memory storage of firing of the feature detector.

(2) Such facilitation is not restricted to the specific features defining the item as a sensory category, but spreads to the motor and memory outputs of detectors connected to the specific features through connections set up by a process of past learning or association. We must assume that such a process of linkage through past learning or association is responsible for the formation of arbitrary categories (letters, numbers, etc.) which are then represented by separate detectors.

(3) Analogous to the process of facilitation, there is also a process of inhibition, acting again on the output to the motor system and memory of feature detectors which the subject had been asked to disregard, and summing algebraically with the process of facilitation.

(4) As with facilitation, such inhibition also spreads to detectors connected through past association. These may also signal the presence of members of arbitrary categories.

How would this hypothesis work? Suppose the letter $A$ is to be detected. Then the motor and memory connections of the letter A will be facilitated, as will all those of the detectors linked with the letter A detector. These will be feature detectors, signaling various components of the shape of the letter, whose joint discharge triggers the letter detector. (Such discharge is not facilitated or inhibited. Only the discharge of the connections to motor and memory circuits is.) Further, there will be other letter detectors on the same level (B, C, D, etc.) whose motor and memory output will be facilitated. Finally, there will be facilitation of the motor and memory connection of detectors signaling, for example, the category of letters, to which the letter A has been connected through past association. Such facilitation will flow from the A detector through pathways established during the associative process. An analogous process of inhibition will occur if the letter $\mathbf{A}$ is to be disregarded. If inhibition exceeds facilitation beyond a certain level in the motor output pathway, excitation from the feature detector cannot pass down the pathway and no response is triggered. However, when inhibition exceeds facilitation by a lesser amount, a reduction of excitation flowing down the pathway will result. There will be a slowing of response depending on the amount of net inhibition.

Let us now apply this hypothesis to the category effect. Suppose the subject is asked to detect the letter A while disregarding digits. First, the motor and memory outputs of the letter A detector will be facilitated along with that of those detectors connected to it through past association. These will be feature detectors, signaling the components of the shape, other letters detectors, and the letter-category detector. Second, the motor and memory outputs of the digit detectors will be inhibited, with inhibition of the same outputs also of the detectors associated with the digits (shape, other digits, digit category). As there is relatively little overlap or associative connection between the digit category and the letter category, and digits and letters, there will be relatively little cancellation of facilitation and inhibition on the motor and memory outputs of the detectors subserving them. However, there is a very high degree of overlap among the component shapes of the letter and the digits. Consequently, the detectors signaling such components will have their motor and memory outputs simultaneously facilitated and inhibited. However, the motor and memory outputs from the A letter detector and the letter-category detector will be highly facilitated, little inhibition reaching these detectors. This will lead to a short latency of response. On the other hand, the digit and digit-category detectors will have their motor and memory outputs inhibited almost exclusively, and as a result, memory storage after they have been fired will be poor. Further, if another letter instead of $A$ is presented when $A$ is expected, there will be a high probability that a response will be initiated. There will be virtually no inhibition on the motor and memory outputs of that letter detector and the letter-category detector. As a result, there will be net facilitation of the motor and memory outputs of these detectors, making a response probable, though tending to be slower. Gleitman and Jonides (1976) have experimental data bearing on this question. Unfortunately, as they themselves state, two of their three experiments could be confounded with response set, which would tend to reduce the difference between the reaction times between the to-be-detected item and another belonging to the same category inserted on a catch trial. In spite of this and the small $\mathrm{N}$, some 
trend in the direction expected by the present hypothesis is evident. In a third experiment, where the response set interpretation can be excluded, no reaction time data are quoted. On the Jonides and Gleitman hypothesis (1972), the reaction time on the catch trials should be the same. On the present hypothesis, there should be a high probability of response but its latency should be higher.

The second case to be explained in the category effect is what happens when an item is to be detected from among items in the same category. For instance, the subject is instructed to detect an A among other letters. The response to the other letters is always to be withheld. As we believe that the constituent shape feature detectors for digits and letters are drawn from a completely overlapping set, there will tend to be an averaging of facilitation and inhibition on the motor and memory outputs as the number of items increases. On the other hand, the supraordinate category to which all the items on the display now belong will tend to have its motor outputs inhibited. Further, some inhibition will be relayed from the items to be disregarded to the to-bedetected item by virtue of past association. By the same token, facilitation will be relayed from the to-be-detected item to the to-be-disregarded items. This means that the reaction time to the $A$ will be slower, but the memory of the to-be-disregarded items will be better than in the first case ( $A$ among digits) discussed above. This accords with Gleitman and Jonides' (1976) experimental data.

In the third case, where the same item is a member of two categories (as in the oh-zero phenomenon), the verbal instructions will selectively facilitate different groups of detectors. When the instruction is to detect a zero among a group of letters, the motor and memory outputs of the "zero" detector, the number-category detector and those triggered by other digits will be facilitated and the motor and memory outputs of the letter detectors and the lettercategory detector will be inhibited. The overall situation will be very similar to that of the first case (A among digits).

When the instruction is to detect an "oh" among a group of letters, the "oh" detector, the motor and memory outputs of the letter-category detector, and those detectors triggered by other letters will be facilitated. On the other hand, because other letters are to be disregarded, the letter-category detector and other letter detectors will also have their motor and memory outputs inhibited to a greater or lesser extent. Such inhibition will also subtract from the facilitation of the "oh" detector.

Consequently, in terms of the total pattern of facilitation and inhibition, the situation will be the same when "oh" is to be detected among letters as when any other letter is to be detected among letters. We would therefore expect a longer reaction time when we ask the subject to detect an "oh" among letters than when we ask the subject to detect a "zero"' of the same shape among letters.

Finally, on the Jonides-Gleitman theory, the reaction time to select an "oh" or another letter from among digits should be the same if the subject is asked to find a letter (unspecified). However, on the present theory, it should take longer to find an item with a membership in both categories (such as an "oh") than to find an item belonging only to one category (such as an A).

\section{REFERENCES}

Brand, J. Classification without identification in visual search. Quarterly Journal of Experimental Psychology, 1971, 23, 178-186.

Deutsch, J. A., \& Deutsch, D. Attention: Some theoretical considerations. Psychological Review, 1963, 70, 80-90.

Egeth, H., Jonides, J., \& W ALl, S. Parallel processing of multielement displays. Cognitive Psychology, 1972, 3, 674-698.

Gleitman, H., \& Jonides, J. The cost of categorization in visual search: Incomplete processing of target and field items. Perception \& Psychophysics, 1976, 20, 281-288.

INGLING, N. W. Categorization: A mechanism for rapid information processing. Journal of Experimental Psychology, 1972, 94, 239-243.

Jonides, J., \& Gleitman, H. A conceptual category effect in visual search: $O$ as a letter or as a digit. Perception \& Psychophysics, 1972, 12, 457-460.

Jonides, J., \& GLeItman, H. The benefit of categorization in visual search: Target location without identification. Perception \& Psychophysics, 1976, 20, 289-298.

(Received for publication March 23, 1977; accepted A pril 4, 1977.) 